(2) Open Access Full Text Article

\title{
Improving access to shared decision-making for Hispanics/Latinos with inadequately controlled type 2 diabetes mellitus
}

This article was published in the following Dove Press journal:

Patient Preference and Adherence

30 April 2015

Number of times this article has been viewed

\author{
Jaime A Davidson' \\ Aracely Rosales ${ }^{2}$ \\ Alicia C Shillington ${ }^{3}$ \\ Robert A Bailey ${ }^{4}$ \\ Chris Kabir ${ }^{3}$ \\ Guillermo E Umpierrez ${ }^{5}$ \\ 'Touchstone Diabetes Center, \\ University of Texas Southwestern \\ Medical Center, Dallas, TX, \\ USA; ${ }^{2}$ Rosales Communications, \\ Philadelphia, PA, USA; ${ }^{3} \mathrm{EPI}-\mathrm{Q}$, Inc., \\ Oakbrook, IL, USA; ${ }^{4} \mathrm{Health}$ Economics \\ and Outcomes Research, Janssen \\ Scientific Affairs, LLC, Raritan, NJ, \\ USA; ${ }^{5}$ Division of Endocrinology \\ and Metabolism, Emory University, \\ Atlanta, Georgia, GA, USA
}

Purpose: To describe the cultural and linguistic adaptation and Spanish translation of an English-language patient decision aid (PDA) for use in supporting shared decision-making in Hispanics/Latinos with type 2 diabetes mellitus (T2DM), a group at a high risk for complications.

Patients and methods: A steering committee of endocrinologists, a primary care physician, a certified diabetes educator, and a dietician, each with extensive experience in providing care to Hispanics/Latinos was convened to assess a PDA developed for English-speaking patients with T2DM. English content was reviewed for cultural sensitivity and appropriateness for a Hispanic/Latino population. A consensus-building process and iterative version edits incorporated clinician perspectives. The content was adapted to be consistent with traditional Hispanic/ Latino cultural communication precepts (eg, avoidance of hostile confrontation; value for warm interaction; respect for authority; value of family support for decisions). The PDA was translated by native-speaking individuals with diabetes expertise.

Results: The PDA underwent testing during cognitive interviews with ten Spanish-speaking Hispanics/Latinos with T2DM to ensure that the content is reflective of the experience, understanding, and language Hispanic/Latino patients use to describe diabetes and treatment. Content edits were made to assure a literacy level appropriate to the audience, and the PDA was produced for online video dissemination.

Conclusion: High-quality, well-developed tools to facilitate shared decision-making in populations with limited access to culturally sensitive information can narrow gaps and align care with individual patient preferences. A newly developed PDA is available for shared decisionmaking that provides culturally appropriate treatment information for inadequately controlled Hispanics/Latinos with T2DM. The impact on the overall health of patients and care management of T2DM requires further study.

Keywords: patient decision aid, language adaptation, Hispanic, decision making, type 2 diabetes

\section{Introduction}

Hispanics/Latinos constitute the largest minority ethnic group in the United States. ${ }^{1,2}$ Hispanics/Latinos have higher documented rates of chronic illnesses, including cardiovascular disease, diabetes, asthma, and cancer than the general population. ${ }^{3-7}$ Specifically, diabetes poses a major public health concern for this population, who experience a greater risk of diabetes-associated morbidity and mortality versus the general US population, ${ }^{8,9}$ and poorer glycemic control compared to non-Hispanic/ non-Latino whites. ${ }^{3,10-12}$

The Triple Aim, a framework for health care improvement developed by the Institute for Healthcare Improvement, is an approach endorsed by the medical community to optimize health care delivery. It is being adopted by provider organizations to improve
Correspondence: Alicia C Shillington EPI-Q, Inc., 1315 West 22nd Street, Suite 410, Oak Brook, IL 60523, USA $\mathrm{Tel}+\mathrm{I} 6306052393$

Fax +l 6305705506

Email alicia.shillington@epi-q.com 
quality and reduce the cost of suboptimal outcomes. A key objective of the Triple Aim is to identify disparities, understand and stratify the needs of "populations of concern", and activate those populations with patient-centered strategies to improve health. ${ }^{13}$ In US Hispanics/Latinos, differences in access to quality health care, socioeconomic and cultural factors, genetics and epigenetics may explain some of the disparity in health outcomes. ${ }^{3,5,14,15}$ Approximately 43\% of Hispanics/Latino immigrants arrive with only a grade school education. Many of the immigrants have limited English knowledge, and greater than 95\% speak only Spanish at home, inhibiting Englishlanguage acquisition; only a very small percentage demonstrate proficient health literacy. ${ }^{16,17}$ In one of the largest assessments carried out to date, a survey of 19,000 households oversampled for Hispanics/Latinos, only $4 \%$ of Hispanic/Latinos in the National Assessment of Adult Literacy (NAAL) demonstrated "proficient" health literacy, and 41\% scored "below basic". ${ }^{16}$ Patients in poverty and those with inadequate health literacy report generally poorer health relative to more health-literate counterparts. ${ }^{18,19}$ As Hispanics/Latino immigrants tend to maintain their connection to the Spanish language and culture, patient-centered health promotion initiatives designed to address disparities and promote health activation must be developed and delivered in a linguistically appropriate as well as culturally sensitive manner. ${ }^{20,21}$

In the most recent Standards of Medical Care in Diabetes, the American Diabetes Association (ADA) emphasizes an individualization of care using a systematic patient-centered approach for the optimal management of patients with type 2 diabetes mellitus (T2DM). This approach involves health care professionals partnering with patients to choose among guideline-recommended medications consistent with patient values and life circumstances. Quality of care is determined by delivered care consistent with guidelines. ${ }^{22}$ Shared decision-making is a patient-centered, collaborative process that enables patients and their providers to make health decisions together while taking into account the best scientific evidence available as well as the patient's preferences. ${ }^{23}$ Patient decision aids (PDAs) are tools that communicate information on treatment options and facilitate patient choice that is consistent with values and preferences, serving as a cornerstone of high-quality care. ${ }^{24}$ However, existing PDAs are not widely available for Hispanic/Latino audiences. Given that health providers and health educators can do little to influence economic and language disparities, it is important to create culturally and linguistically aligned PDAs that respect the socio-demographics, cultural values, beliefs, and practices of the intended audience.
A well-designed, evidence-based PDA is available in English to assist decision-making for intensified medical treatment in patients with T2DM who exhibit inadequate glycemic control on metformin. ${ }^{25}$ The aid presents unbiased information regarding the options available for treatment intensification along with potential benefits and detriments of each option, and it facilitates patients' clarification of values relative to the treatment decision. It also provides a mechanism for patients to discuss the decision with family members and other trusted individuals, and communicates patients' preferences to their physicians.

The objective of the work reported in this manuscript was to cross-culturally and linguistically adapt the English PDA for Spanish-speaking Hispanics/Latinos with T2DM not currently controlled on metformin. Literal translation of materials developed for an English-speaking audience without cross-cultural and linguistic adaptation may lead to information that is not relevant and understandable. In this work, we describe our process of adaptation that encompasses not only translation from English to Spanish, but also crosscultural adaptation of the material to be reflective of the experience and culture of a Hispanic/Latino audience.

\section{Patients and methods}

A PDA was previously developed in English with input from clinicians, an expert on shared decision-making, and patients. ${ }^{25}$ The intent is to assist those with uncontrolled T2DM treated with metformin to make better decisions about intensifying therapy. The target users of the original PDA are English-speaking adults who are currently taking metformin and have inadequately controlled T2DM, and have reached the decision point in their care provision in which their physicians are considering additional medication.

The PDA was designed in alignment with the ADA recommendations to prepare patients, health care and health education professionals for decision-making by providing unbiased information on medication options including potential risks and benefits, eliciting and communicating patient preferences to clinicians, and promoting shared decisionmaking during a clinician consultation. In order to provide unbiased information, a framework was used in which each medication class discussed included an explanation of potential disadvantages and advantages for the following domains: mode of administration, effect on weight, efficacy in reducing blood sugar levels, potential side-effects, and cost. By providing the opportunity for patients to improve their knowledge on multiple treatment options available and self-reflect on the importance of values related directly 
to their personal decision-making, the PDA is designed to prepare patients for enhanced discussion with their health care provider on selecting treatment options that are aligned to individual preferences.

To cross-culturally adapt the PDA for a Hispanic/Latino audience, a Latino Cultural Adaptation and Review Committee (LCARC) consisting of two endocrinologists, a primary care physician, a diabetes educator, and a dietician, each providing care to Hispanics/Latinos, was convened. Also included in the process was a Hispanic/Latino health literacy expert. A systematic and well-defined process ${ }^{26}$ was used by the expert committee for cross-cultural adaptation, which was conducted between November 2013 and July 2014 (Figure 1). The adaptation from the English version was developed with the goal of aligning with the Ottawa Decision Support Framework. The framework includes: meeting decisional needs to minimize uncertainty, improve knowledge, and clarify expectations and values; improving decisional quality by making choices that are informed, values based, and timely; and providing decision support through education, portrayal of factual information, values clarification, and communication guidance. $^{27}$

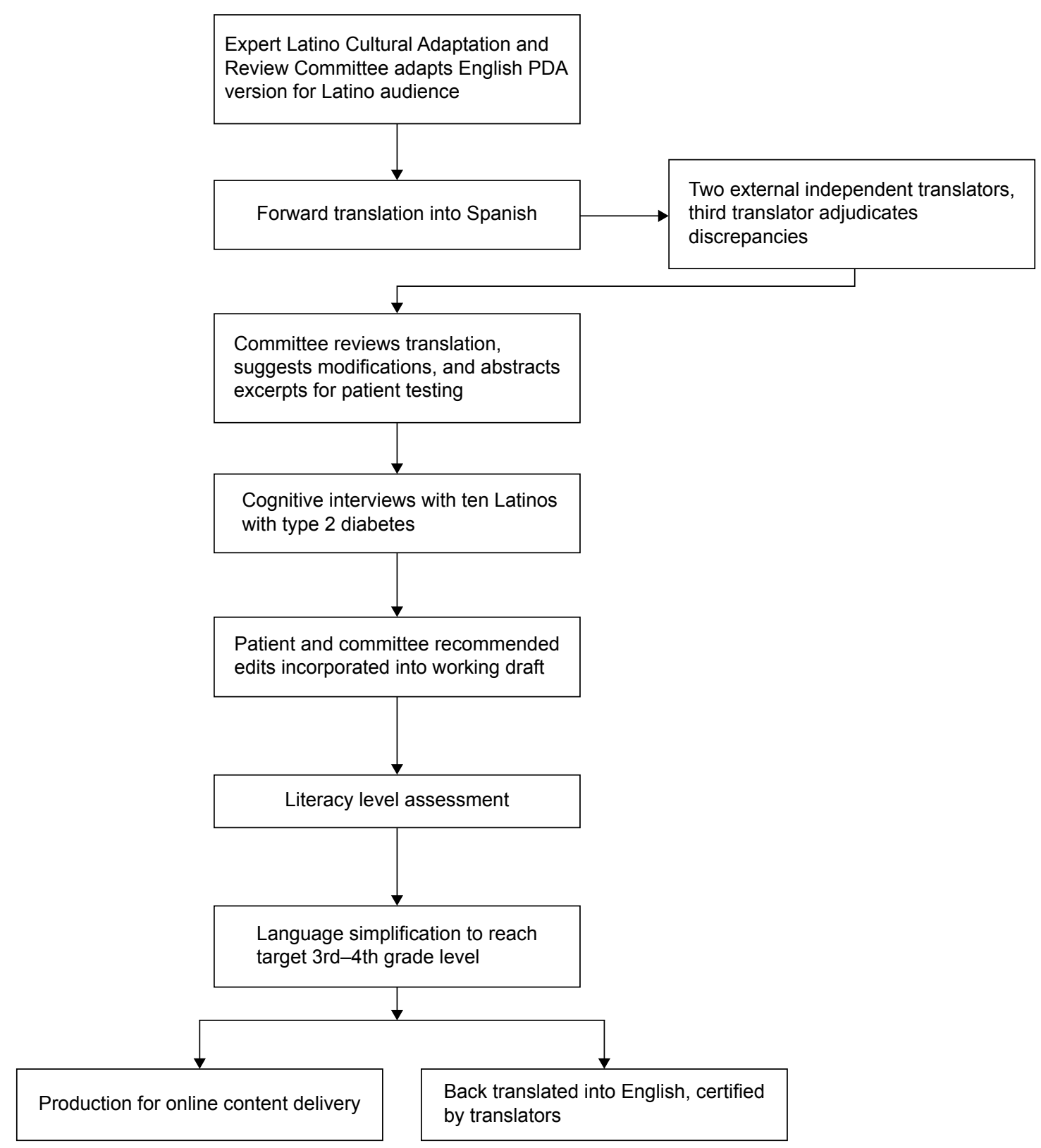

Figure I Cross-cultural adaptation process. Abbreviation: PDA, patient decision aid. 
The adaptation process involved an initial review of the English version by the health care providers to adapt the content to be specifically relevant to Hispanics/Latinos. Reviewers were asked to assess and modify the content to be congruent with cultural themes common among Hispanics/ Latinos including: 1) simpatia - politeness and the avoidance confrontational language; 2) personalismo - the value of warm personal interaction; 3 ) respeto or jeraquismo - respect for authority figures, usually including health care providers; 4) familismo - relationship and inclusion of extended family in health care decisions; and 5) fatalismo - superstitions and the belief in fate. ${ }^{28}$ Care was taken to adapt, modify, or remove colloquialisms and abbreviations unfamiliar to Hispanics/Latinos, and to use examples regarding self-care behaviors (eg, diet, self-care, and family support) consistent with Hispanic/Latino culture. The committee members also added content with the purposes of dispelling myths and misconceptions common to Hispanic/Latino individuals with diabetes (eg, insulin is addicting, my sugar is in God's hands - nothing I do matters, only orange juice can be used to raise blood sugar in a hypoglycemic episode). A consensus-building process with the committee, including teleconferences and iterative version edits, was undertaken to incorporate Hispanic/Latino clinician perspectives into the English version. Each iteration of the adaptation was reviewed and edited by the multidisciplinary steering committee until consensus was achieved on the final content for translation into Spanish.

Upon completion of the initial adaptation of the English version, the content was translated into Spanish using a standard forward translation process. Two independent translators unfamiliar with the PDA and its development process individually forward translated the PDA script content into Spanish. The versions between the two translators were compared, and where differences existed, a third expert linguist adjudicated the discrepancies to create a harmonized script. The harmonized script was then reviewed by the LCARC prior to obtaining patient input. The committee members discussed, suggested modifications, and then abstracted excerpts where disagreements in translation existed. Also abstracted were the translations of more complex clinical concepts (eg, insulin resistance, pancreatitis as a side effect of medication). These excerpts were incorporated into a discussion guide for obtaining patient input via cognitive interviews.

Excerpts that were tested in cognitive interviews conducted by phone in Spanish with ten Hispanics/Latinos with T2DM are available here http://www.dovepress.com/ get_supplementary_file.php?f=80552.pdf. Interviewees were randomly selected through a nationally represented panel of respondents (Harris Interactive) who spoke Spanish and were included based upon the following criteria confirmed in the interview: 1) age of 18 years or older; 2) currently being treated with metformin for T2DM; 3 ) fluent in Spanish; and 4) were notified by their health care provider that they require additional medication to help control their blood sugar. The occurrence of a discussion between a health care provider and a patient on additional medication for T2DM was used as a surrogate indicator for inadequate glycemic control.

The intent of the interviews was to ensure that the content in the final PDA was relevant and reflective of the experience, understanding, and language Hispanics/Latinos use to describe their diabetes and treatment. Edits were suggested by patients, iteratively discussed by the expert committee, and subsequently incorporated into the working version. Given the disparity in health literacy among Hispanics/Latinos and as the original English version was developed at the 7th-8th grade level, we then examined the content for Spanish literacy level using the Fry-based formula (Gilliam-Peña-Mountain). No current guidelines exist regarding the recommended literacy level for content delivered by video. However, given the educational disparity among Hispanics/Latinos, the committee recommended a final series of iterative edits to simplify the language to the $3 \mathrm{rd}-4$ th grade level. Upon completion of the lower literacy level version, the content underwent a final review by the Hispanic/Latino endocrinologists prior to content production for online delivery.

\section{Results}

Ten Hispanic/Latino patients with diabetes (five male, five female) ranging in age from $31-66$ (mean $=48$ years) reviewed excerpts of the translation provided ahead of a phone interview and were asked to comment on the clarity of content and consistency with their diabetes experience. Countries of origin represented and the number of participating patients were: Argentina, Ecuador, Guatemala (two), Mexico (two), Nicaragua, Puerto Rico (two), and Spain. All but one patient were current full-time residents in the US, nine of ten had at least some high school education, and one was a college graduate. A professional interpreter conducted patient interviews in Spanish using open-ended questions. Interviews lasted 30 minutes on average and probing was used to elicit feedback during the interviews.

Response to PDA content was consistently positive based upon unstructured feedback. Comments suggested that the content was understandable and relevant. All ten patients reported comprehension of the material regardless of age, 
sex, or country of origin, providing some recommendations for edits to include culture-specific wording changes.

Literacy testing indicated that the content was delivered verbally at the equivalent of a 7th-8th grade level, and upon consideration of the committee, it was determined that a 3 rd- 4 th grade level would have a broader audience. A further round of adaptation focused solely on achieving this level. An example of language simplified from the 8th grade level to the 4th grade level when referring to the timing of adding another agent to metformin includes:

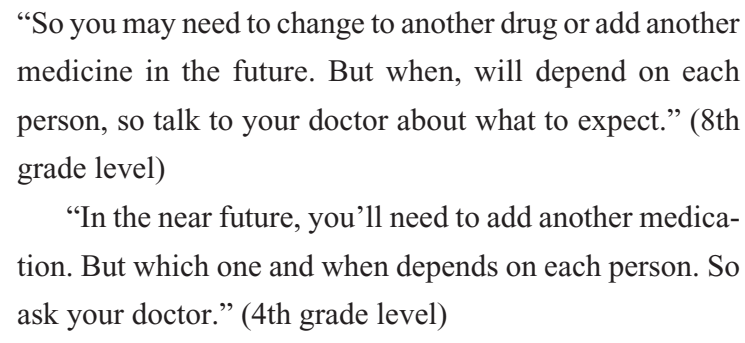

"In the near future, you'll need to add another medication. But which one and when depends on each person. So ask your doctor." (4th grade level)

Upon the lower literacy level adaptation, the content was back-translated into English to assure congruence with the original English version, finalized, and produced for dissemination in an online video format.

\section{Discussion}

Shared decision-making has been gaining support among professional societies and policy makers in the US, as manifested by the recent ADA recommendations. However, PDAs in the US are currently available primarily for an Englishspeaking audience. Shared decision-making requires patients to be provided with the descriptions of available treatment options, and presented with the potential benefits and detriments of each option. ${ }^{29}$ High-quality, well-developed tools can facilitate shared decision-making; ${ }^{30}$ however, shared decision-making may be challenging for patients, especially Hispanics/Latinos due to cultural, language, and literacy influences. There is a need to provide access to culturally sensitive PDAs to help improve health knowledge, narrow disparity gaps, and facilitate care consistent with values important to Hispanics/Latinos.

This manuscript provides a description of the systematic process used to cross-culturally adapt a PDA for Hispanics/ Latinos, including input from an interdisciplinary group of health care professionals, patients, and linguistic experts. Our PDA is developed to help Hispanic/Latino patients understand their options, clarify their own values, and communicate preferences in a culturally sensitive manner.

The cross-cultural adaptation process outlined here considers input from clinicians and patients who are likely to use and benefit from a PDA for a Hispanic/Latino population with diabetes. The process we describe can be used to adapt information on complex topics to suit individuals at risk for disparities and related negative outcomes in a culturally sensitive manner, and to increase knowledge and support for decision-making. Patients responded positively to the PDA content and recognized the value of having information available related to medication options for T2DM when treatment intensification is required. Based on this feedback, it is likely that this PDA will support the T2DM medication decision-making process among Hispanics/Latinos.

Clinicians and diabetes educators treating Hispanics/ Latinos recognize the important role that family (familismo) plays in diabetes treatment and self-management. ${ }^{31}$ The product of this adaptation process is a PDA that can be delivered online wherever internet access is possible. This allows the flexibility for patients to include family members in the use of the PDA and the decision-making process. The PDA encourages dialogue between patients, families, and health care professionals. It provides the opportunity to empower patients and family with information about treatment options as they interact with clinicians typically viewed as respected people in authority and as trusted sources of information by Hispanics/Latinos. ${ }^{32}$

The need to include Hispanic/Latino patient cultural perspectives in developing or adapting PDAs was confirmed during our process. Our findings are consistent with previous qualitative assessment of views toward medical decisionmaking in Hispanics/Latinos, and indicate that they are more likely than Whites to discuss attitudes including positive outlook, faith, religion, and family support. ${ }^{32}$ Our initial adaptation steps included a multidisciplinary team of experts well-grounded in the care of Hispanics/Latinos with diabetes. Steps included modifying the content to include themes important to this population. However, patient interviews identified parts of content requiring further modification to maintain a positive tone, and to honor respectful communication with health care providers.

Shared decision-making relies on a patient being willing to accept a role in that decision, jointly with a clinician. However, not all patients want to participate in shared decision-making. Hispanics/Latinos may wish to be less participatory than non-Hispanic/Latinos ${ }^{33,34}$ and may prefer to have physicians make decisions on their behalf. ${ }^{35}$ In addition to the ADA patient-centered care mandate, the Institute of Medicine's "Crossing the Quality Chasm" indicates that physicians should encourage patients to participate in their health care decision-making to the extent that they are 
willing. ${ }^{36}$ Although Hispanics/Latinos may be less willing to participate in shared decision-making, the majority express that they prefer to have at least some role in discussing and understanding treatment options, and they should be encouraged to do so. Hispanics/Latinos and non-Hispanic/ Latinos alike demonstrate information-seeking behavior from trusted sources when faced with a health care decision. ${ }^{37}$ In the absence of other sources of information, Hispanics/ Latinos' trusted sources are likely to be word of mouth of family and personal relations. ${ }^{32}$ In our PDA, we encourage patients to share the information in the PDA with trusted individuals who, although well-intended, may be sources of inaccurate or misinformation, and as such, the aid can help assure accurate information is obtained.

Our PDA has several areas that warrant further investigation. The assessment included only clinicians who were involved in the PDA cross-cultural adaptation process. It has not yet been assessed in clinicians beyond the development team. Only a small number of patients were included in cognitive interviews, and because of the geographic distribution of the interviewees, interviews could not be conducted face to face. These individuals were carefully selected to reflect the target audience of future users; however, literacy levels of patients in the pilot may have been higher than some end-users once implemented. Future work may include piloting the PDA with a wider Hispanic/Latino audience and validating effects on knowledge, self-efficacy, and decisional conflict (in particular, the certainty in making the best choice, level of feeling informed and supported in decision-making, and clarity about personal values for the choice's risks and benefits). In addition, further assessment of the PDA may include acceptability measures pertaining to the way in which information is presented and its perceived usefulness in preparation for decision-making within the user's cultural experience. It is not clear how well Hispanics/Latinos will accept an impersonal internet-based video, given that their culture places particularly high value on close interpersonal relationships. While reliance on the internet for content delivery may facilitate wide content delivery, it will not address the needs of those lacking internet access. This may be relevant for a group more likely to be financially constrained than the general US population. However, when internet and computer access is available, online PDAs have the advantages of being more accessible to users who wish to view the information at a comfortable setting outside of the doctor's office, at a time that is convenient for them, and watch with family members or others who may be important in their decision-making process. A validation study is required to understand the effectiveness and acceptability of the PDA in this specific population.

\section{Conclusion}

This initiative supports the importance of developing culturally sensitive tools for Hispanic/Latino patients with T2DM, as disparities exist. A newly developed PDA culturally appropriate for Hispanics/Latinos with poorly controlled T2DM is available to facilitate shared decision-making between clinicians and patients (https://www.diabetesdecisionaid.com). The PDA was developed and adapted using a systematic approach involving a panel of clinical experts and patients. A further study on the impact of the use of the culturally-adapted PDA is needed.

\section{Acknowledgment}

The authors wish to acknowledge Anne Farley, RN MSM, for her assistance in the adaptation process. This research and report were developed with funding from Janssen Scientific Affairs, LLC.

\section{Disclosure}

RAB is an employee of Janssen Scientific Affairs, LLC and a Stockholder in Johnson and Johnson. The authors report no other conflicts of interest in this work.

\section{References}

1. Camarota SA. Immigrants in the United States, 2010: A Profile of America's Foreign-Born Population. Washington DC: USA: Center for Immigration Studies; 2012.

2. Lollack L. The Foreign-Born Population in the United States. Population Characteristics; 2001. Available from: http://www.census.gov/ prod/2000pubs/p20-534.pdf

3. Centers for Disease C Prevention. Prevalence of diabetes and impaired fasting glucose in adults - United States, 1999-2000. MMWR Morb Mortal Wkly Rep. 2003;52(35):833-837.

4. Daviglus ML, Talavera GA, Avilés-Santa ML, et al. Prevalence of major cardiovascular risk factors and cardiovascular diseases among Hispanic/ Latino individuals of diverse backgrounds in the United States. JAMA. 2012;308(17):1775-1784.

5. Graham EA, Jacobs TA, Kwan-Gett TS, Cover J. Health services utilization by low-income limited English proficient adults. J Immigr Minor Health. 2008;10(3):207-217.

6. Pugh JA, Stern MP, Haffner SM, Eifler CW, Zapata M. Excess incidence of treatment of end-stage renal disease in Mexican Americans. Am J Epidemiol. 1988;127(1):135-144.

7. Resnick HE, Foster GL, Bardsley J, Ratner RE. Achievement of American Diabetes Association clinical practice recommendations among U.S. adults with diabetes, 1999-2002: the National Health and Nutrition Examination Survey. Diabetes Care. 2006;29(3): 531-537.

8. Karter AJ, Ferrara A, Liu JY, Moffet HH, Ackerson LM, Selby JV. Ethnic disparities in diabetic complications in an insured population. JAMA. 2002;287(19):2519-2527.

9. Services USDoH, Human. Healthy People 2010: Understanding and Improving Health. 2nd ed. Washington DC: USA: 2000. 
10. Getaneh A, Light LS, Brillon DJ, et al. Diabetes control among Hispanics in the action to control cardiovascular risk in diabetes trial journal of general internal medicine. J Gen Intern Med. 2012;27(11): 1499-1505.

11. Oza-Frank R, Chan C, Liu K, Burke G, Kanaya AM. Incidence of type 2 diabetes by place of birth in the Multi-Ethnic Study of Atherosclerosis (MESA). J Immigr Minor Health. 2013;15(5):918-924.

12. Selvin E, Parrinello CM, Sacks DB, Coresh J. Trends in prevalence and control of diabetes in the United States, 1988-1994 and 1999-2010. Ann Intern Med. 2014;160(8):517-525.

13. Berwick DM, Nolan TW, Whittington J. The triple aim: care, health, and cost. Health Aff. 2008;27(3):759-769.

14. Introduction and literature review. In: Smedley BD, Stith AY, Nelson AR, editors. Unequal Treatment: Confronting Racial and Ethnic Disparities in Health Care (with CD). Washington, DC: The National Academies Press; 2003:29-79.

15. Williams MV, Parker RM, Baker DW, et al. Inadequate functional health literacy among patients at two public hospitals. JAMA. 1995;274(21): 1677-1682.

16. Kutner MA, United States Department of Education, National Center for Education Statistics. The Health Literacy of America's Adults: Results from the 2003 National Assessment of Adult Literacy. Washington, DC: United States Department of Education, National Center for Education Statistics; 2006.

17. Flores G. Language barriers to health care in the United States. $N$ Engl J Med. 2006;355(3):229-231.

18. Berkman ND, Sheridan SL, Donahue KE, Halpern DJ, Crotty K. Low health literacy and health outcomes: an updated systematic review. Ann Intern Med. 2011;155(2):97-107.

19. Bennett IM, Chen J, Soroui JS, White S. The contribution of health literacy to disparities in self-rated health status and preventive health behaviors in older adults. Ann Fam Med. 2009;7(3):204-211.

20. Rosal MC, Ockene IS, Restrepo A, et al. Randomized trial of a literacysensitive, culturally tailored diabetes self-management intervention for low-income latinos: latinos en control. Diabetes Care. 2011;34(4): $838-844$

21. Betancourt JR, Green AR, Carrillo JE, Ananeh-Firempong O 2nd. Defining cultural competence: a practical framework for addressing racial/ethnic disparities in health and health care. Public Health Rep. 2003;118(4):293-302.

22. American Diabetes Association. Standards of Medical care in Diabetes 2015. 2015; 38(suppl 1):S1-S80.

23. Informed Medical Decisions Foundation. What is shared decision making? 2014. Available from: http://www.informedmedicaldecisions.org/ what-is-shared-decision-making/. Accessed August 22, 2014.
24. Montori VM, LeBlanc A, Buchholz A, Stilwell DL, Tsapas A. Basing information on comprehensive, critically appraised, and up-to-date syntheses of the scientific evidence: a quality dimension of the International Patient Decision Aid Standards. BMC Med Inform Decis Mak. 2013;13(suppl 2):S5.

25. Shillington AC, Col N, Bailey RA, Jewell MA. Development of a patient decision aid for type 2 diabetes mellitus for patients not achieving glycemic control on metformin alone. Patient Prefer Adherence. 2015;9:609-617.

26. Beaton DE, Bombardier C, Guillemin F, Ferraz MB. Guidelines for the process of cross-cultural adaptation of self-report measures. Spine. 2000;25(24):3186-3191.

27. O’Connor A. Ottawa Decision Support Framework to Address Decisional Conflict; 2006. Available from: https://decisionaid.ohri.ca/docs/ develop/ODSF.pdf. Accessed October 15, 2014.

28. Villa ML, Cuellar J, Gamel N, Yeo G. Aging and Health: Hispanic American Elders. 2nd ed. Palo Alto, CA: Stanford Geriatric Education Center; 1993.

29. Elwyn G, O'Connor AM, Bennett C, et al. Assessing the quality of decision support technologies using the International Patient Decision Aid Standards instrument (IPDASi). PLoS One. 2009;4(3):e4705.

30. Legare F, Stacey D, Turcotte S, et al. Interventions for improving the adoption of shared decision making by healthcare professionals. Cochrane Database Syst Rev. 2014;9:CD006732.

31. Denham SA, Ware LJ, Raffle H, Leach K. Family inclusion in diabetes education: a nationwide survey of diabetes educators. Diabetes Educ. 2011;37(4):528-535.

32. Katz JN, Lyons N, Wolff LS, et al. Medical decision-making among Hispanics and non-Hispanic whites with chronic back and knee pain: a qualitative study. BMC Musculoskelet Disord. 2011;12:78.

33. Xu KT, Borders TF, Arif AA. Ethnic differences in parents' perception of participatory decision-making style of their children's physicians. Med Care. 2004;42(4):328-335.

34. Patel SR, Bakken S. Preferences for participation in decision making among ethnically diverse patients with anxiety and depression. Community Ment Health J. 2010;46(5):466-473.

35. Levinson W, Kao A, Kuby A, Thisted RA. Not all patients want to participate in decision making. A national study of public preferences. J Gen Intern Med. 2005;20(6):531-535.

36. Institute of Medicine. Crossing the Quality Chasm: A New Health System for the 21st Century. Washington, DC: 2001.

37. Livingston G, Minushkin S, Cohn D. Hispanics and health care in the united states: access, information and knowledge. A Joint Pew Hispanic Center and Robert Wood Johnson Foundation Research Report, Washington, DC; 2008.
Patient Preference and Adherence

\section{Publish your work in this journal}

Patient Preference and Adherence is an international, peer-reviewed, open access journal that focuses on the growing importance of patient preference and adherence throughout the therapeutic continuum. Patient satisfaction, acceptability, quality of life, compliance, persistence and their role in developing new therapeutic modalities and compounds to optimize

\section{Dovepress}

clinical outcomes for existing disease states are major areas of interest for the journal. This journal has been accepted for indexing on PubMed Central. The manuscript management system is completely online and includes a very quick and fair peer-review system, which is all easy to use. Visit http://www. dovepress.com/testimonials.php to read real quotes from published authors. 